\title{
THE CARBON STAR PHENOMENON
}

Edited by ROBERT F. WING
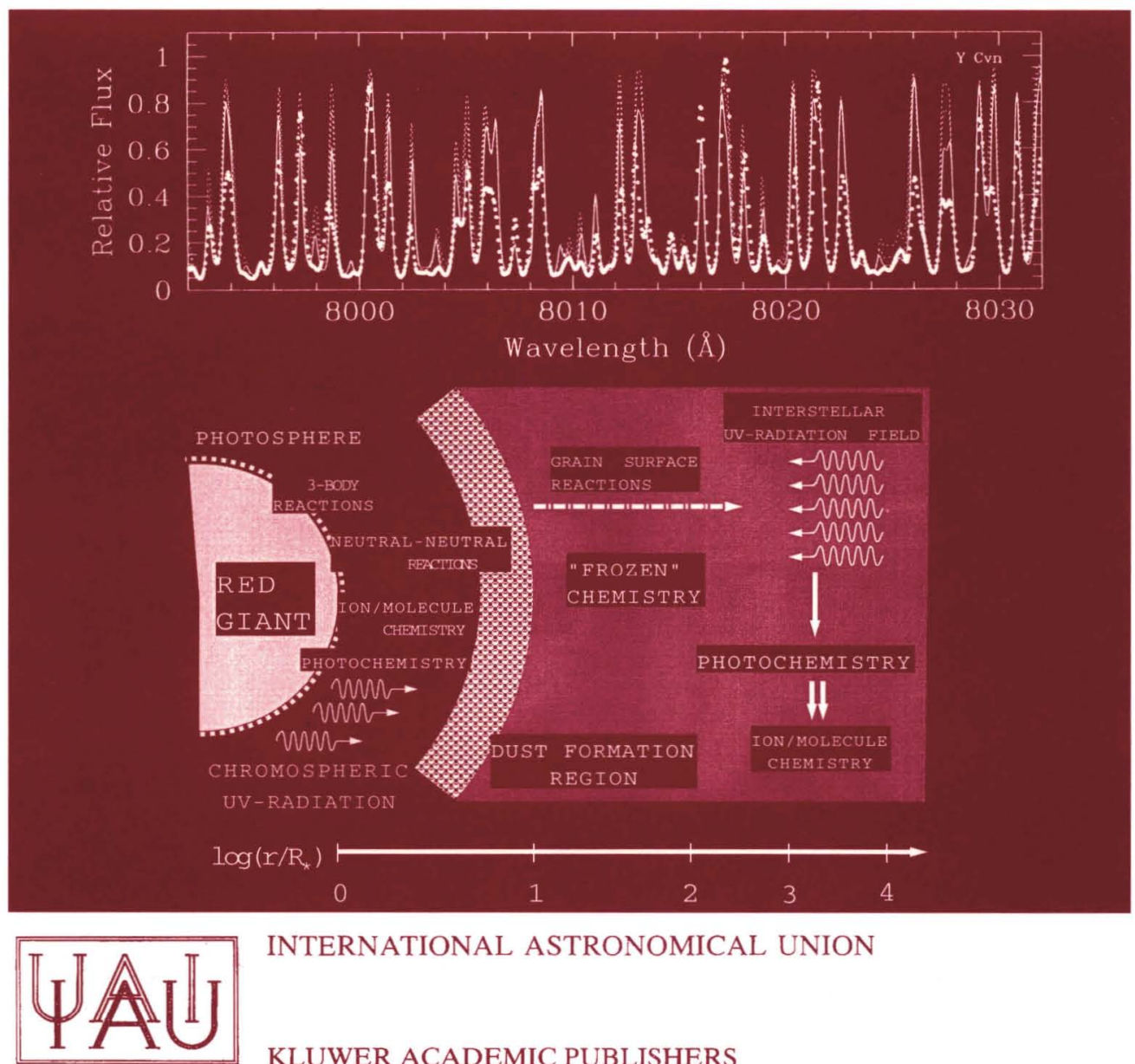

INTERNATIONAL ASTRONOMICAL UNION

KLUWER ACADEMIC PUBLISHERS 


\section{THE CARBON STAR PHENOMENON}




\title{
THE CARBON STAR PHENOMENON
}

PROCEEDINGS OF THE 177TH SYMPOSIUM OF THE INTERNATIONAL ASTRONOMICAL UNION, HELD IN ANTALYA, TURKEY, MAY 27-31, 1996

\author{
EDITED BY \\ ROBERT F. WING \\ Astronomy Department, \\ The Ohio State University, \\ Columbus OH, U.S.A.
}

\section{KLUWER ACADEMIC PUBLISHERS}

DORDRECHT / BOSTON / LONDON

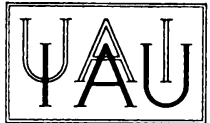


A C.I.P. Catalogue record for this book is available from the Library of Congress.

ISBN 0-7923-6346-9

\author{
Published on behalf of \\ the International Astronomical Union \\ by \\ Kluwer Academic Publishers, P.O. Box 17, 3300 AA Dordrecht, The Netherlands. \\ Sold and distributed in North, Central and South America \\ by Kluwer Academic Publishers, \\ 101 Philip Drive, Norwell, MA 02061, U.S.A. \\ In all other countries, sold and distributed \\ by Kluwer Academic Publishers, \\ P.O. Box 322, 3300 AH Dordrecht, The Netherlands.
}

Printed on acid-free paper

\author{
All Rights Reserved \\ (C) 2000 International Astronomical Union
}

No part of the material protected by this copyright notice may be reproduced or utilized in any form or by any means, electronic or mechanical, including photocopying, recording or by any information storage and retrieval system, without written permission from the publisher.

Printed in the Netherlands. 


\section{CONTENTS}

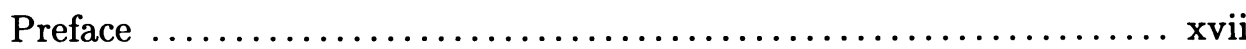

Tribute to Nüzhet Gökdoğan $\ldots \ldots \ldots \ldots \ldots \ldots \ldots \ldots \ldots \ldots \ldots \ldots \ldots \ldots \ldots \ldots \ldots \ldots$

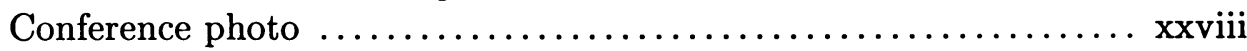

List of participants $\ldots \ldots \ldots \ldots \ldots \ldots \ldots \ldots \ldots \ldots \ldots \ldots \ldots \ldots \ldots \ldots \ldots \ldots \ldots \ldots$

\section{Introduction}

Introductory Remarks: Watch the Details $\ldots \ldots \ldots \ldots \ldots \ldots \ldots \ldots . \ldots$

How to Deal with Unexpected Scientific Results

William P. Bidelman (read in absentia)

What Theorists Think They Know about AGB Stars $\ldots \ldots \ldots \ldots \ldots .7$ John C. Lattanzio

\section{Session I - Surveys}

Deep IV-N and Yellow-Red Spectral Surveys for Carbon Stars ...... 13 O. M. Kurtanidze and M. G. Nikolashvili

Space Distribution of Carbon Stars in Our Galaxy ............. 21

K. Noguchi, Z. Qian, J. Sun, G. Wang, J. Wang and Y. Rao

Understanding the Elusive Dwarf Carbon Star ............... 27

Paul J. Green

A Proper-Motion Search for Dwarf Carbon and S Stars .......... 37

D. J. MacConnell, R. L. Williamson II and W. J. Roberts

Large Magellanic Cloud Carbon Stars: Reading the Rosetta Stone of Stellar Evolution .................................. 41

Jay A. Frogel and Edgardo Costa

Surveys for Carbon Stars in External Galaxies ................. 51

Marc Azzopardi

A Photometric and Spectroscopic Survey of AGB Stars in M31 ..... 59

James P. Brewer, Harvey B. Richer and Dennis R. Crabtree

\section{Session II - Models and Abundance Determinations}

Model Atmospheres for Normal and Peculiar Red Giant Stars ...... 71 Bertrand Plez 
vi

Quantitative Analysis of Carbon Isotope Ratios in N-, SC-, and

J-type Carbon Stars $\ldots \ldots \ldots \ldots \ldots \ldots \ldots \ldots \ldots \ldots \ldots \ldots \ldots . \ldots \ldots$

K. Ohnaka and T. Tsuji

${ }^{12} \mathrm{C} /{ }^{13} \mathrm{C}$ Ratios and Li Abundances in Low-Mass C Stars .......... 89

Carlos Abia and Jordi Isern

Carbon and Hydrocarbon Molecules in Very Cool White Dwarfs .... 97

Turgut Aslan and Irmela Bues

The $s$-Process in the Yellow Symbiotic AG Draconis ............ 103

Katia Cunha, Verne V. Smith and Alain Jorissen

The Chromospheres of Carbon Stars

Donald G. Luttermoser

\section{Session III - Observed Spectra and Energy Distributions}

Spectral Characteristics of RV Tauri Stars $\ldots \ldots \ldots \ldots \ldots \ldots \ldots \ldots . \ldots 117$

Sunetra Giridhar

Applications of Narrow-Band Photometry in the Study of Peculiar

Red Giants ..................................... 127

Robert F. Wing

Spectral Classification of Carbon-Peculiar G Stars

Tuba Koktay and R. F. Garrison

Mass-losing AGB Stars in the LMC ...................... 145

Jacco Th. van Loon, Albert A. Zijlstra, Patricia A. Whitelock,

Cecile Loup and L. B. F. M. Waters

\section{Session IV - Variability}

Trend Analysis of 51 Carbon Long-Period Variables ............. 155

Janet Akyüz Mattei and Grant Foster

Comparison of Mean Light Curve Parameters of M, S and C Mira and Semi-Regular Variable Stars Using 75 Years of AAVSO Data . . 165

Marie-Odile Mennessier, Hichame Boughaleb and

Janet A. Mattei

The Variability of R, N, and C Stars from HIPPARCOS and AAVSO

Data ........................................ 171

Michel Grenon, Janet A. Mattei, Laurent Eyer and Grant Foster

Infrared Light Curves of Carbon-Rich Variables ............... 179

Patricia Whitelock 
R CrB: Photometric Evidence Regarding the Nature of its Pulsation, and a Putative Connection between Pulsation and Deep Declines .. 191 J.D. Fernie

The Pulsations and Evolution of AGB Stars in the Large Magellanic Cloud

Z. G. Gong and Y. Li

\section{Session V - Post-AGB Stars}

Post-AGB Variables and Stellar Mass-Loss $\ldots \ldots \ldots \ldots \ldots \ldots \ldots \ldots 207$ M. W. Feast

Lines of Circumstellar $\mathrm{C}_{2}, \mathrm{CN}$, and $\mathrm{CH}^{+}$in the Optical Spectra of

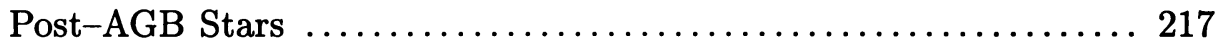

Eric J. Bakker, David L. Lambert and Ewine F. Van Dishoeck

Chemical Composition and Evolution of Post-AGB Stars ......... 225

M. Parthasarathy

Optical Spectroscopic Monitoring of the Carbon-Rich Post-AGB Star HD 56126: Pulsation and Shock Waves

Agnès Lèbre, Nicolas Mauron, Denis Gillet and

Dominique Barthès

Collimated Outflow from Stars: The Planetary Nebula Abell $78 \ldots 245$ Paris Pişmiş (read in absentia)

\section{Session VI - Binarity}

The Role of Binaries in the Carbon Star Phenomenon ........... 249 Robert D. McClure

Barium Stars and Tc-Poor S Stars: Binary Masqueraders within the

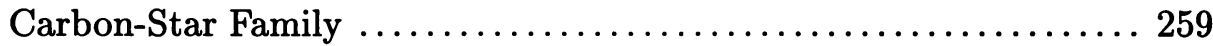

A. Jorissen and S. Van Eck

Binarity among Barium Dwarfs and CH Subgiants: Will They

Become Barium Giants?

Pierre North, Alain Jorissen and Michel Mayor

The Chemical Composition and Orbital Parameters of Barium Stars . 277 Laimons Začs

Binary "Post-AGB" Stars 285

Hans Van Winckel, Christoffel Waelkens and

Laurens B.F.M. Waters 
viii

Light and Velocity Variability of Post-AGB Stars $\ldots \ldots \ldots \ldots \ldots \ldots 293$

Bruce J. Hrivnak and Wenxian $\mathrm{Lu}$

The Case for S Star Binaries ........................... 299

Thomas B. Ake

Session VII - Mass-Loss, Winds, and Formation of Dust Grains

Inferring Mass Loss Rates for Cool Luminous Stars from

High-Resolution GHRS Spectra $\ldots \ldots \ldots \ldots \ldots \ldots \ldots \ldots \ldots \ldots \ldots . \ldots \ldots$

J. L. Linsky, G. M. Harper, J. Valenti, P. D. Bennett and

A. Brown

Dust Formation in Stellar Photospheres .................... 313

The Case of Carbon Stars from Dwarfs to AGB Stars

Takashi Tsuji

Dust-Driven Winds of Rotating Carbon Stars ............... 325

Ernst A. Dorfi and Susanne Höfner

Nucleating Dust in Carbon-Rich AGB Stars ................. 331

I. Cherchneff

Grain Formation in the Winds of Cool Red Giant Stars .......... 337

E. Sedlmayr and J.M. Winters

Carbon Star Dust from Meteorites

Uffe Gråe Jørgensen and Anja C. Andersen

\section{Session VIII - Circumstellar Shells}

Circumstellar Dust around M, S and C Stars ................ 361

Irene R. Little-Marenin

Observations of Mass Loss and Circumstellar Matter around Cool

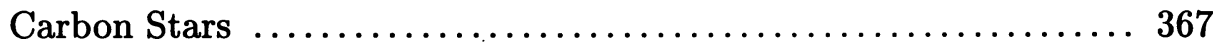

T. Lloyd Evans

Dynamical Models of Circumstellar Dust Shells around Long-Period Variables

A. J. Fleischer, J. M. Winters and E. Sedlmayr

Modelling the Spectral Energy Distributions of AGB Stars in the LMC

M. A. T. Groenewegen, J. Th. van Loon, P. A. Whitelock,

P. R. Wood and A. A. Zijlstra 
IR Emission from Dusty Winds - Scaling and Self-Similarity

Properties ... 391

Moshe Elitzur and Željko Ivezić

Dust Emission from IRC +10216 399

Željko Ivezić and Moshe Elitzur

Session IX - Circumstellar Emission and Environment

Molecular Optical and Infrared Emission from the Red Rectangle ... 407 Carriers of Diffuse Circumstellar, Nebular and Interstellar Bands

T. H. Kerr, J. R. Miles, M. E. Hurst, R. E. Hibbins and

P. J. Sarre

Scattered Light from Envelopes around N-type Stars ............ 409

Bengt Gustafsson, Kjell Eriksson, Dan Kiselman, Nils Olander,

Hans Olofsson and Hugo E. Schwarz

The Neutral Envelopes around AGB and Post-AGB Objects ...... 413

Their Structure and Kinematics

H. Olofsson

Extended Dust Shells around Carbon Stars in the Infrared and in

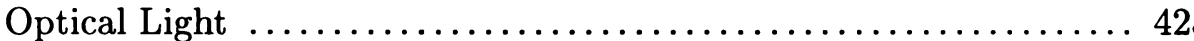

Hideyuki Izumiura, L. B. F. M. Waters, T. de Jong, C. Loup

and $O$. Hashimoto

Asymmetries around Luminous Red Variables

Antonio Mário Magalhães and Kenneth H. Nordsieck

\section{Session X - Nucleosythesis and Evolution}

Heavy-Element Nucleosynthesis in AGB Stars ................ 443 Verne V. Smith

Nucleosynthesis in Intermediate-Mass Stars

John C. Lattanzio, Cheryl A. Frost, Robert C. Cannon and

Peter R. Wood

Fluorine Production in Asymptotic Giant Branch Stars

N. Mowlavi, A. Jorissen and M. Arnould

Carbon Stars in the Early-AGB Stage of Evolution 463

Ju. L. Frantsman

From the Tip of the AGB Towards a Planetary: A Hydrodynamical

Simulation

D. Schönberner, M. Steffen, J. Stahlberg, K. Kifonidis and

T. Blöcker 


\section{Session XI - Galactic Chemical Evolution}

Carbon Stars and Nucleosynthesis in Galaxies ................. 481 Bengt Gustafsson and Nils Ryde

\section{Session XII - Observing Facilities}

Variability Studies with International Networks ............... 499 François R. Querci and Monique Querci

A New Optical Observatory in Turkey .................... 507 Zeki Aslan, Selim O. Selam and Akif Esendemir

\section{Abstracts of Posters}

(ordered alphabetically by first author)

$V$ and $R$ Observations of Two Carbon Stars: UX Dra and RY Dra .. 515 Hasan Ak, Berahitdin Albayrak, Zeki Aslan, Osman Demircan, Zekeriya Müyesseroğlu, Sacit Özdemir and Kutluay Yüce

Unusual Light Curves of Some Carbon Stars ................. 516 Andrejs Alksnis

Modelling the M-S-C Giants Spectral Sequence .............. 517 France Allard, Peter H. Hauschildt, David R. Alexander, Martin Cohen and Gordon C. Augason

Carbon Isotope Ratios in Carbon Stars of the Galactic Halo ....... 518 Wako Aoki and Takashi Tsuji

Carbon Stars in Open Clusters ............................. 519 Bernhard Aringer

The SiO Molecule in the Atmospheres of Cool AGB Stars 520 Bernhard Aringer, Uffe G. Jørgensen, Stephen R. Langhoff and Josef Hron

The Eddington Limit, Radiative Instabilities and the Declines of

R Coronae Borealis Stars ............................ 521 Martin Asplund

Modelling of Carbon-Rich Stars with Far Infrared Flux Excess ..... 522 Stefano Bagnulo, Gerry Doyle, Chris Skinner and

Vincenzo Andretta

Selective Depletion of Elements in Stellar Atmospheres: A Unified Picture? ..................................... 523

Eric J. Bakker, Guillermo Gonzalez and David L. Lambert 
How to Make Carbon Stars: A New Approach to Model Boundaries of Convective Regions ................................ 524

T. Blöcker, F. Herwig, D. Schönberner and M. El Eid

Opacities for Carbon Dwarfs and M Dwarfs .................. 525

Aleksandra Borysow and Uffe Gråe Jørgensen

A Kinematic Survey of Carbon Stars in the Small Magellanic Cloud.. 526

Russell Cannon, Barry Croke, Despina Hatzidimitriou and

David Morgan

Mid-Infrared Silicate Variation in Long-Period, Oxygen-Rich

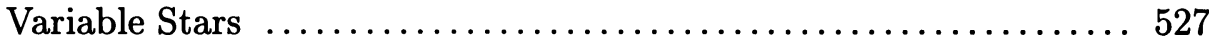

M. J. Creech-Eakman and R.E. Stencel

LMC/SMC Outer Halo Carbon Star Survey: Radial Velocities of

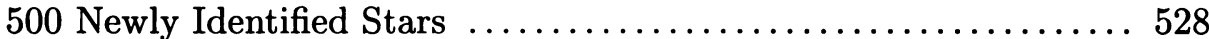

Serge Demers, W.E. Kunkel and M.J. Irwin

The Backwarming Effect and Carbon Stars ................. 529

Dimitri N. Doikov and Ekaterina M. Doikova

Proposal for a Photometric System for the Classification of

Carbon Stars ....................................... 530

Uldis Dzervitis

Carbon to Oxygen Abundance Ratios in the Atmospheres of Carbon

Stars of the Orion and Perseus Galactic Arms ................ 531

I. Eglitis and M. Eglite

Condensation of $\mathrm{SiC}$ in Circumstellar Dust Shells of C-Rich

Red Giants ..................................... 532

Andreas Gauger, John J. Keady and Erwin Sedlmayr

New Results from the Modeling of the Shell around IRC $+10216 \ldots 533$

M. A. T. Groenewegen

$J H K$ Photometry of AGB Stars in the SMC 534

M. A. T. Groenewegen and J. A. D. L. Blommaert

Frequency Sampling for Radiative Transfer Calculations in

Cool Stars

Christiane Helling and Uffe Gråe Jørgensen

Macro-Molecules in Model Atmospheres .................... 536

Christiane Helling, Uffe Gråe Jørgensen, Bertrand Plez and

Hollis R. Johnson

Molecular Hydrogen in the Circumstellar Shells of Carbon Stars ... 537 Kenneth Hinkle and John Keady 
xii

Atmospheric Dynamics and Dust-Driven Winds of Carbon Stars ... 538 Susanne Höfner and Ernst A. Dorfi

The Shape of Silicate Features in Semiregular and Mira Variables ... 539 Josef Hron, Bernhard Aringer and Franz Kerschbaum

FG Sge as a New-Born Carbon Star ..................... 540

Takashi Iijima

Experimental Gas-Phase Spectroscopy of Carbon Molecular

Structures and Diffuse Interstellar Bands .................. 541

J. Janča, M. Plonka, M. Šolc and M. Vetešník

The Population of Red Giant Stars in Globular Clusters of the

Fornax Dwarf Galaxy ............................. 542

Uffe Gråe Jørgensen and Raul Jimenez

Synthetic JHK Colors for M Dwarfs, M Giants, and Carbon Stars ... 543 Uffe Gråe Jørgensen and Robert F. Wing

Near-Infrared Spectroscopy of High Galactic Latitude Carbon Stars -

They Might Be Giants? ............................. 544

Richard R. Joyce

A Simulation for a Carbon Star ......................... 545

Gülçin Kandemir and Cem Güçlü

On the Nature of Irregular Variables of Type Lb .............. 546

Franz Kerschbaum, Peter Habison and Josef Hron

Comparison of C-Rich Mira, Semiregular, and Irregular Variables ... 547 Franz Kerschbaum, Peter Habison, Rita Loidl, Hans Olofsson and Josef Hron

The Chemical Composition of the Halo Mira V CrB $\ldots \ldots \ldots \ldots \ldots . . \ldots 48$ Tônu Kipper and Uffe Gråe Jørgensen

A $200 \mathrm{~km} / \mathrm{sec}$ Molecular Wind in the Carbon Star V Hya ......... 549 Gillian Knapp, Alain Jorissen and Ken Young

Formation of Core-Mantle Type Grains Consisting of a SiC Core and

a Carbon Mantle in Circumstellar Envelopes of Carbon Stars .... 550

Takashi Kozasa and Hisato Sogawa

Open and Globular Cluster Ages Using Theoretical Isochrones ..... 551 İbrahim Küçük

Tien Shan Astronomical Observatory 552 Kenesken Kuratov

Statistics of Carbon Stars in the Galaxy Omar M. Kurtanidze 
A Close Association of Three Carbon Stars in the Direction of M92 .. 554 O. M. Kurtanidze and M. G. Nikolashvili

Near IR $(J H K)$ Observations of Selected Carbon Stars ............ 555 Vladimir P. Kuz'kov

A Comparison between the Mira-OH/IR and the Carbon-Rich AGB

Sequences ....................................... 556

Jacques R. D. Lépine

Interferometric Molecular Line Observations of RW LMi

Michael Lindqvist, Robert Lucas, Hans Olofsson, Fredrick

Larsen, Alain Omont, Kjell Eriksson and Bengt Gustafsson

Are There Silicate - S Stars?

Irene R. Little-Marenin

Classification of Dust Emission Features in Carbon Stars .......... 559

Irene R. Little-Marenin, Gregory C. Sloan and Stephan D. Price

Synthetic Spectra for Carbon-Rich Long-Period Variables ......... 560

Rita Loidl, Bernhard Aringer, Uffe Jørgensen, Susanne Höfner and Josef Hron

Circumstellar Envelopes of Peculiar and Normal J-Type Stars ..... 561

S. Lorenz Martins

Modelling of $\mathrm{OH} / \mathrm{IR}$ Dust Envelopes ..................... 562

S. Lorenz Martins and F.X. de Araújo

Southern Carbon Stars Found on Near-IR Objective-Prism Plates .. 563 D. Jack MacConnell

Absolute Magnitude and Kinematics of Barium Stars 564

Marie-Odile Mennessier, Ana Gómez, Xavier Luri, Suzanne

Grenier, Louis Prévot, Jordi Torra and Francesca Figueras

The Overtone Spectrum of Molecular Hydrogen and Methane in the

Visible: Recent Measurements ........................ 565

Michael Mickelson, Lee Larson, Lars English and David Ferguson

Multiwavelength Photometric Observations of Northern Carbon Stars 566

Anatoly S. Miroshnichenko, Kenesken S. Kuratov, Željko Ivezić

and Moshe Elitzur

AFGL 4106: Proto-Planetary Nebula or Post-Red Supergiant?

Frank Molster, Jacco van Loon, Rens Waters and

Hans Van Winckel

The Detection of Low-Luminosity Carbon Stars in the Leo II and 
Fornax Dwarf Galaxies .............................. 568

Gérard Muratorio and Marc Azzopardi

High-Resolution Coudé-Echelle Spectrometer for the 1.5-m Kazan University Telescope at the Turkish National Observatory ....... 569 Faig Musaev and Ilfan Bikmaev

Spectroscopic Analysis of Single-Lined Spectroscopic Binaries with Unseen Companions .............................. 570

Faig Musaev, Ilfan Bikmaev and Laimons Začs

Radiative Transfer and Dynamics of Stellar Outflows 571

Nathan Netzer

A Determination of the C/M5+ Ratio in the Galactic Plane ....... 572 Maria G. Nikolashvili

On the Molecular Structure of Circumstellar Envelopes Surrounding C Stars ........................................ 573

A. Beate C. Patzer, Jan Martin Winters and Erwin Sedlmayr

Maser Mapping of Red Supergiants and the Onset of Bipolar Outflow . 574 Anita M.S. Richards, Jeremy A. Yates and R. James Cohen

The Peculiar Object IRAS 06088+1909 .................. 575

A. Richichi, G. Calamai, F. Lisi, B. Stecklum, T. Herbst and

E. Thamm

Observations, Assignments and Profiles of $\mathrm{SiC}_{2}$ Absorption and Emission Bands in Carbon Stars ....................... 576

Peter J. Sarre, Mark E. Hurst and Tom Lloyd Evans

The Dust around Cool Stars ............................ 577

Irakli Simonia and Tsitsino Simonia

Observations of the $11 \mu \mathrm{m}$ Silicon Carbide Feature in Carbon Star Shells ........................................ 578

Angela K. Speck, M. J. Barlow and C. J. Skinner

Carbon- and Oxygen-Rich Stars in the IRAS Two-Color Diagram:

Results from Hydrodynamical Models of AGB Winds ........... 579

M. Steffen, R. Szczerba, A. Men'shchikov and D. Schönberner

Energy Distribution in the Spectra of Carbon Stars ............. 580 Janis-Imants Straume

On Carbon Star Evolution in the IRAS Two-Color Diagram ....... 581 Ryszard Szczerba, Matthias Steffen and Kevin Volk

Carbon Stars in the Galactic Halo 582

Ed J. Totten and Michael J. Irwin 
Infrared Observations of Peculiar Carbon Stars ................ 583 Ana Ulla, Peter Thejll, Tônu Kipper and Uffe Gråe Jørgensen

The Frequency of Extrinsic and Intrinsic S Stars in the Henize

Sample ........................................ 584

Sophie Van Eck, Alain Jorissen, Michel Mayor, Stephane Udry and Michel Burnet

New Input Data for Synthetic AGB Evolution ................ 585

J. Wagenhuber

Silicon and Sulphur Chemistry in the Inner Envelopes of Carbon

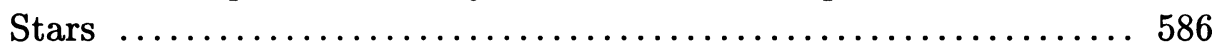

Karen Willacy and Isabelle Cherchneff

Synthetic Colors of Carbon Stars ...................... 587

Walter Windsteig, Ernst A. Dorfi, Susanne Höfner, Josef Hron

and Franz Kerschbaum

Temperatures of Peculiar G-Type Stars from Narrow-Band

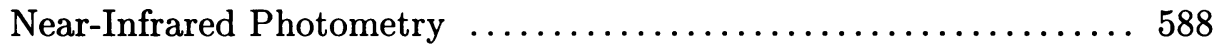

Robert F. Wing, Robert F. Garrison and Tuba Koktay

A Photometric Search for Dwarf Carbon Stars ................ 589

Robert F. Wing and D. Jack MacConnell

Optical Appearance of Dynamical Models for Circumstellar Dust

Shells around Long-Period Variables: AFGL 3068 ........... 590

Jan Martin Winters, Axel J. Fleischer, Thibaut Le Bertre

and Erwin Sedlmayr

Envelope Pulsations of a $1 M_{\odot}$ AGB Star During Thermal Pulses ... 591 A. Ya'ari, Y. Tuchman and J. Wagenhuber

Motions of Carbon Stars ............................. 592

Cahit Yeşilyaprak, Zeki Aslan, Orhan Gölbaşı and

Tuncay Özdemir

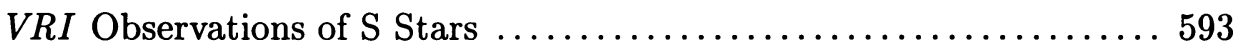

Sandra B. Yorka and Tracy L. Huard

Liquid and Solid Carbon Particles in Cool White Dwarf Atmospheres 594 Victor Zubko

\section{Indexes}

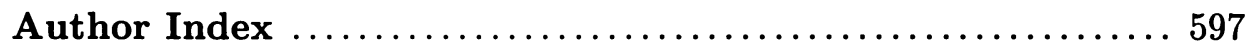

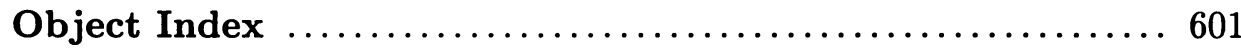

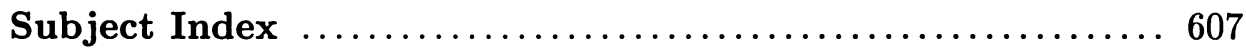


xvi

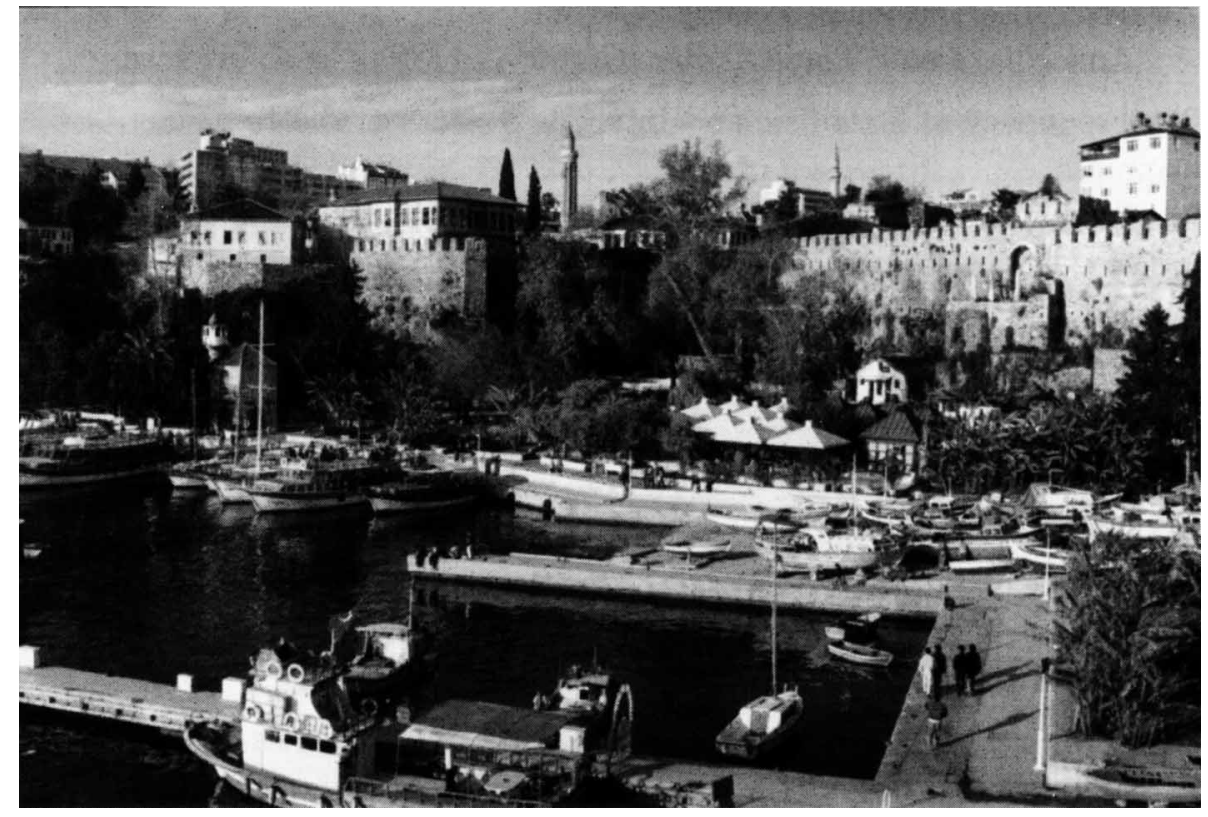

View of the Antalya harbor. Visible in the rear center is the 13 th century fluted brick Seljuk minaret which has become a landmark of the city. 